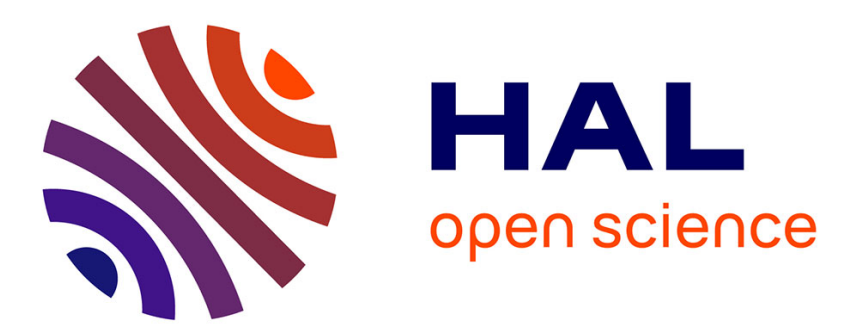

\title{
Hierarchy of LMI conditions for the stability analysis of time delay systems
}

Alexandre Seuret, Frédéric Gouaisbaut

\section{To cite this version:}

Alexandre Seuret, Frédéric Gouaisbaut. Hierarchy of LMI conditions for the stability analysis of time delay systems. Systems and Control Letters, 2015, 81, pp.1-7. 10.1016/j.sysconle.2015.03.007 . hal-01065142

\section{HAL Id: hal-01065142 \\ https://hal.science/hal-01065142}

Submitted on 18 Sep 2014

HAL is a multi-disciplinary open access archive for the deposit and dissemination of scientific research documents, whether they are published or not. The documents may come from teaching and research institutions in France or abroad, or from public or private research centers.
L'archive ouverte pluridisciplinaire HAL, est destinée au dépôt et à la diffusion de documents scientifiques de niveau recherche, publiés ou non, émanant des établissements d'enseignement et de recherche français ou étrangers, des laboratoires publics ou privés. 


\title{
Hierarchy of LMI conditions for the stability analysis of time delay systems
}

\author{
A. Seuret ${ }^{a, b}$ F. Gouaisbaut ${ }^{a, c}$ \\ ${ }^{\text {a }}$ CNRS, LAAS, 7 avenue du Colonel Roche, 31077 Toulouse, France. \\ (e-mail: aseuret,fgouaisb@laas.fr). \\ ${ }^{\mathrm{b}}$ Univ de Toulouse, LAAS, F-31400 Toulouse, France \\ ${ }^{\mathrm{c}}$ Univ de Toulouse, UPS, LAAS, F-31400, Toulouse, France.
}

\begin{abstract}
Assessing stability of time-delay systems based on the Lyapunov-Krasovskii functionals has been the subject of many contributions. Most of the results are based, first, on the design of more and more involved class of functionals and, finally, on the use of the famous Jensen's inequality. In contrast with this design process, the present paper aims at providing a generic set of integral inequalities which are asymptotically non conservative and then to design functionals driven by these inequalities. The resulting set of stability conditions forms a hierarchy of LMI which is competitive with the most efficient existing methods (delay-partitioning, discretization and sum of squares), in terms of conservatism and of complexity. Finally, some examples show the efficiency of the method.
\end{abstract}

Key words: Bessel inequality, Jensen's inequality, stability analysis, time-delay systems, Lyapunov-Krasovskii functionals

\section{Introduction}

In the past two decades, a large number of papers appeared on stability of time delay systems [11, 22, 29]. Several research lines have been explored with success, regarding the case of a linear system with a single delay. A first idea consists in the study of the characteristic equation associated with the delay system (see [11, 29] and references therein). Even though these methods provide necessary and sufficient condition for stability, extensions to time-varying delays or robustness issue lead to unavoidable difficulties. Another interesting method relies on the modeling of the original delay system as a closed loop between a nominal system and the delay operator, which is embedded into a suitable uncertainty. Then robust analysis tools like Small gain Theorem [11, 32], IQCs [16] or Quadratic separation [7] allow to conclude.

On the other side, one of the most fruitful fields of research in this area relies on the exhibition of LyapunovKrasovskii Functionals (LKF) for functional differential equations. Unlike the case of ordinary differential equations, the introduction of delay hugely complicates the task and was therefore the source of many theoretical developments $[4,19]$. For a single and constant delay, a Lyapunov functional candidate, called complete LKF
[17] leads to a necessary and sufficient stability condition. Nevertheless, the parameters, which compose the complete LKF, i.e. the matrix functions depend on the solution of a differential equation with boundary conditions and is recognized to be numerically difficult to handle especially for high dimensional systems [21]. A number of investigations then turns to approximating the parameters of the complete LKF. Hence, the problem has been then partially solved by considering a discretization scheme proposed originally by [11]. The interval of integration for the quadratic forms are partitioned into smaller delay domain and the structure of the matrix functions are chosen to be piecewise continuous within these intervals. It results a numerically tractable Linear Matrix Inequality (LMI) problem, which is efficient on some examples. More recently, this approximation method has been improved by considering polynomial-like parameter of arbitrary degree. By considering polynomial relaxations, the original problem of finding an LKF may be recasted into an SOS optimization scheme [25]. All these two approximation methods provide with only sufficient conditions, but potentially tends to be necessary if the discretization step or the polynomial degree are refined [24].

On the other side, many papers have been concentrated on the research of a simpler LKF whose parameters are 
optimized via an LMI setup. The goal is often to study the trade-off between computational complexity of the resulting LMI and the pessimism induced by the simplification of the LKF. In the literature, two mainstream methods, often coupled in practice, have been followed. The first one proposed by $[1,8,13,28,31,30]$ is to consider a simpler structure for the LKF than the previously mentioned complete LKF which is applied to some higher dimensional comparison systems. The additional degrees of freedom come from the consideration of extrastates like higher derivatives of the states $[1,18]$, delayed states $[8,11]$, integral delayed terms [30]. Nevertheless, it is not so clear that considering some particular extrastate will be more accurate than choosing another. The second one remains in the use of technical lemmas to provide tight inequalities $[14,15,18,23]$. Among them, Jensen inequality has been the core of many interesting improvements [3]. Combined with some delay partitioning approaches [8,13], it leads to efficient criteria. More recently, several papers have employed a new inequality based on Wirtinger Lemma [20, 26, 27]. It allows a significant reduction of the conservatism with a slight modification of the usual LKF. However, it seems, at the first sight, complicated to refine these techniques.

In this paper, we concentrate on this second method and introduce a set of new integral inequalities, which encloses the Jensen inequality [10] and the Improved Wirtinger-based inequality [27] as special cases. These inequalities introduce extra-states functions representing the projection of the delay state $x_{t}$ on the set of Legendre polynomials of degree less than a prescribed upper-bound $N$. Guided by these inequalities and these extra-states, we thus propose a new LKF. The resulting analysis leads to a hierarchy of sufficient LMI stability conditions for the pointwise delays and delay range cases. Finally, some academic examples show the effectiveness of our method even for non trivial examples. In all the cases, the numerical results tend to the analytical bound with a reasonable numerical burden compared to efficient techniques based on the discretization, delay partitioning or SOS optimization.

Notations: Throughout the paper $\mathbb{R}^{n}$ denotes the $n$ dimensional Euclidean space with Euclidian norm $|\cdot|$, $\mathbb{R}^{n \times m}$ is the set of all $n \times m$ real matrices. The notation $P \succ 0$, for $P \in \mathbb{R}^{n \times n}$, means that $P$ is symmetric and positive definite. The sets $\mathbb{S}_{n}$ and $\mathbb{S}_{n}^{+}$represent, the set of symmetric and symmetric positive definite matrices of $\mathbb{R}^{n \times n}$, respectively. The set of continuous functions from an interval $[-h, 0] \subset \mathbb{R}$ to $\mathbb{R}^{n}$ which are, consequently, square integrable is demoted as space $\mathcal{C}$. For any function $f \in \mathcal{C}$, the norm $|f|_{h}$ refers to $\sup _{\theta \in[-h, 0]}|f(\theta)|$. The symmetric matrix $\left[\begin{array}{cc}A & B \\ * & C\end{array}\right]$ stands for $\left[\begin{array}{cc}A & B \\ B^{T} & C\end{array}\right]$. $\operatorname{diag}(A, B)$ stands for the diagonal matrix $\left[\begin{array}{ll}A & 0 \\ 0 & B\end{array}\right]$. Moreover, for any square matrix $A \in \mathbb{R}^{n \times n}$, we define
$\mathrm{He}(A)=A+A^{T}$. The matrix $I$ represents the identity matrix of appropriate dimension. The notation $0_{n, m}$ stands for the matrix in $\mathbb{R}^{n \times m}$ whose entries are zero and, when no confusion is possible, the subscript will be ommitted. For any function $x:[-h,+\infty) \rightarrow \mathbb{R}^{n}$, the notation $x_{t}(\theta)$ stands for $x(t+\theta)$, for all $t \geq 0$ and all $\theta \in[-h, 0]$. The notation $\left(\begin{array}{l}k \\ l\end{array}\right)$ refers to the binomial coefficients given by $\frac{k !}{(k-l) ! ! !}$.

\section{Problem formulation}

Consider a linear time-delay system described by:

$$
\begin{cases}\dot{x}(t)=A x(t)+A_{d} x(t-h), & \forall t \geq 0, \\ x(t)=\phi(t), & \forall t \in[-h, 0],\end{cases}
$$

where $x(t) \in \mathbb{R}^{n}$ is the state vector, $\phi$ is the initial conditions and $A$ and $A_{d}$, are constant matrices. The delay is assumed to be constant. In the context of the stability analysis of such systems using LKF, several types of functionals have been provided in the literature. All of them are composed by the sum of several typical terms [11]. Among them, one of the most relevant components which was introduced in [6] is an integral quadratic term given by

$$
V\left(x_{t}\right)=\int_{-h}^{0} \int_{s}^{0} \dot{x}_{t}^{T}(\theta) R \dot{x}_{t}(\theta) \mathrm{d} \theta \mathrm{d} s
$$

where $\dot{x}_{t}(\theta)=\dot{x}_{t}(t+\theta)$ represents the state of the timedelay system and $h>0$ the delay. The positivity of such functionals is ensured by $R \succ 0$. This class of LyapunovKrasovskii terms has been widely used in the literature mainly because the computation of its time derivative leads to conditions which depend explicitly on the value of the delay $h$. Indeed, differentiating this term with respect to the time variable $t$ leads to

$$
\dot{V}\left(x_{t}\right)=h \dot{x}^{T}(t) R \dot{x}(t)-\int_{-h}^{0} \dot{x}_{t}^{T}(s) R \dot{x}_{t}(s) \mathrm{d} s .
$$

This term is relevant to ensure the negativity of $\dot{V}\left(x_{t}\right)$ because of the negative contribution of the second term. In order to transform (2) into a suitable LMI setup, this integral term should be expressed appropriately in terms of $x_{t}(0)$ and $x_{t}(-h)$. This is made possible thanks to the Jensen inequality (see for instance [11]). Naturally, it is likely to entail some inherent conservatism and several works have been devoted to the reduction of such a gap (see [3] and the references therein). Recently, in $[26,27]$, an alternative inequalities called Wirtingerbased inequalities is employed in order to obtain a more accurate bound for this integral and therefore to reduce the conservatism of the resulting stability conditions. It 
has been shown that an inequality was able to encompass the Jensen's inequality and leads to less conservative results without increasing drastically the computation complexity of the derived LMI's.

The present paper aims at extending the results provided in $[26,27]$ by considering an alternative method based on the Bessel inequality and polynomial approximations. Thanks to this setup, a set of new integral inequalities is provided as showed in following section.

\section{New integral inequalities}

\subsection{Legendre polynomials}

In the following, a brief recall of the Legendre polynomials and their relevant properties is proposed.

Definition 1 The Legendre polynomials considered over the interval $[-h, 0]$ are defined by

$$
\forall k \in \mathbb{N}, \quad L_{k}(u)=(-1)^{k} \sum_{l=0}^{k} p_{l}^{k}\left(\frac{u+h}{h}\right)^{l} .
$$

with $p_{l}^{k}=(-1)^{l}\left(\begin{array}{c}k \\ l\end{array}\right)\left(\begin{array}{c}k+l \\ l\end{array}\right)$.

The set of Legendre polynomials $\left\{L_{k}, k \in \mathbb{N}\right\}$ forms an orthogonal sequence with respect to the inner product:

$$
\langle f, g\rangle=\int_{-h}^{0} f(t) g(t) \mathrm{d} t, \quad \forall f, g \in \mathcal{C} .
$$

Hence, the Legendre polynomials described in Definition 1 satisfy the following properties:

\section{Property 2 P1 Orthogonality:}

$$
\forall(k, l) \in \mathbb{N}^{2}, \quad \int_{-h}^{0} L_{k}(u) L_{l}(u) \mathrm{d} u= \begin{cases}0, & k \neq l \\ \frac{h}{2 k+1}, & k=l\end{cases}
$$

$\boldsymbol{P} 2$ Boundary conditions:

$$
\forall k \in \mathbb{N}, \quad L_{k}(0)=1, \quad L_{k}(-h)=(-1)^{k} .
$$

\section{P3 Differentiation:}

$$
\dot{L}_{k}(u)= \begin{cases}0, & k=0 \\ \sum_{i=0}^{k-1} \frac{(2 i+1)}{h}\left(1-(-1)^{k+i}\right) L_{i}(u), & k \geq 1\end{cases}
$$

\subsection{Bessel-Legendre inequalities}

Based on the Legendre polynomials, the following lemma is derived.
Lemma 3 Let $x \in \mathcal{C}$ and $R \in \mathbb{S}_{n}^{+}$and $h>0$. Define $\mathcal{I}(x, h)$ as follows

$$
\mathcal{I}(x, h):=\int_{-h}^{0} x^{T}(u) R x(u) \mathrm{d} u
$$

Then, the inequality

$$
\mathcal{I}(x, h) \geq \frac{1}{h} \sum_{k=0}^{N}(2 k+1) \Omega_{k}^{T} R \Omega_{k},
$$

holds for all $N \in \mathbb{N}$, where $\Omega_{k}=\int_{-h}^{0} L_{k}(u) x(u) \mathrm{d} u, k=$ $0, \ldots N$.

Proof : Consider a function $x$ in $\mathcal{C}$, a matrix $R$ in $\mathbb{S}_{n}^{+}$ and $h>0$. Define the function $z_{N}$ by

$$
z_{N}(u)=x(u)-\sum_{k=0}^{N} \frac{2 k+1}{h} \Omega_{k} L_{k}(u)
$$

Clearly, $z_{N}$ is in $\mathcal{C}$ and it represents the approximation error between $x$ and its projection to the polynomial set $\left\{L_{k}, k=0, \ldots, N\right\}$ with respect to the inner product (3). The quantity $\mathcal{I}\left(z_{N}, h\right)=\int_{-h}^{0} z_{N}^{T}(u) R z_{N}(u) \mathrm{d} u$ exists and the orthogonal property of the Legendre polynomials yields

$$
\begin{aligned}
\mathcal{I}\left(z_{N}, h\right)= & \int_{-h}^{0} x^{T}(u) R x(u) d u \\
& -2 \sum_{k=0}^{N} \frac{2 k+1}{h}\left(\int_{-h}^{0} L_{k}(u) x(u) d u\right)^{T} R \Omega_{k} \\
& +\sum_{k=0}^{N}\left(\frac{2 k+1}{h}\right)^{2}\left(\int_{-h}^{0} L_{k}^{2}(u) d u\right) \Omega_{k}^{T} R \Omega_{k} .
\end{aligned}
$$

Finally by recalling that $\Omega_{k}=\int_{-h}^{0} L_{k}(u) x(u) \mathrm{d} u$ and $\left(\frac{2 k+1}{h}\right)^{2} \int_{-h}^{0} L_{k}^{2}(u) d u=\frac{2 k+1}{h}$, it yields

$$
\mathcal{I}\left(z_{N}, h\right)=\mathcal{I}(x, h)-\sum_{k=0}^{N} \frac{2 k+1}{h} \Omega_{k}^{T} R \Omega_{k}
$$

Finally, the inequality (5) is obtained by noting that $\mathcal{I}\left(z_{N}, h\right)>0$ since $R \succ 0$. Notice that this proof is related to the Bessel inequality applied to $x \in \mathcal{C}$ equipped with the inner product (3). Hence, inequality (5) will be recalled latter on as Bessel-Legendre (B-L) Inequality. $\diamond$

\subsection{A suitable corollary for the stability analysis of time-delay systems}

As it was mentioned in the introduction, the problem is often to derive a lower bound of $\int_{-h}^{0} \dot{x}^{T}(u) R \dot{x}(u) \mathrm{d} u$. The next corollary addresses this particular problem. 
Corollary 4 Let $x$ be such that $\dot{x} \in \mathcal{C}, R \in \mathbb{S}_{n}^{+}$and $h>0$. Then, the integral inequality

$$
\mathcal{I}(\dot{x}, h) \geq \frac{1}{h} \xi_{N}^{T}\left[\sum_{k=0}^{N}(2 k+1) \Gamma_{N}(k) R \Gamma_{N}(k)\right] \xi_{N},
$$

holds, for all integer $N \in \mathbb{N}$, where

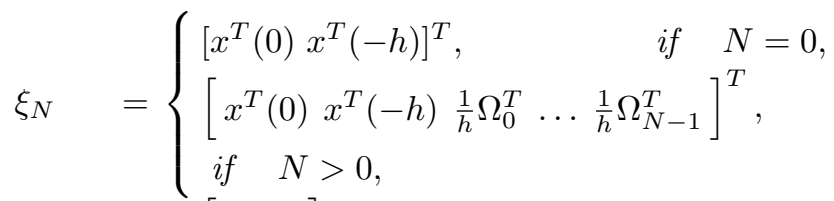

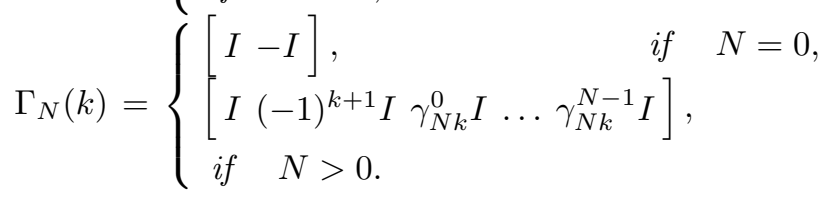

$$
\begin{aligned}
& \gamma_{N k}^{i}= \begin{cases}-(2 i+1)\left(1-(-1)^{k+i}\right), & \text { if } i \leq k, \\
0, & \text { if } i>k .\end{cases}
\end{aligned}
$$

and where $\Omega_{k}$ is defined in Lemma 3.

Proof : According to Lemma 3 to the order $N$, the following inequality holds

$$
\mathcal{I}(\dot{x}, h) \geq \frac{1}{h} \sum_{k=0}^{N}(2 k+1) \tilde{\Omega}_{k} R \tilde{\Omega}_{k},
$$

where $\tilde{\Omega}_{k}=\int_{-h}^{0} L_{k}(u) \dot{x}^{T}(u) \mathrm{d} u$, for all $k=0,1, \ldots, N$. An integration by parts ensures that, for all $k \geq 0$

$$
\tilde{\Omega}_{k}=L_{k}(0) x(0)-L_{k}(-h) x(-h)-\int_{-h}^{0} \dot{L}_{k}(u) x(u) \mathrm{d} u .
$$

Thanks to properties $\mathbf{P} 2$ and $\mathbf{P 3}$ of the Legendre polynomials, the following expression is derived

$$
\tilde{\Omega}_{k}=x(0)-(-1)^{k} x(-h)-\sum_{i=0}^{k-1} \gamma_{N k}^{i} \Omega_{i}=\Gamma_{N}(k) \xi_{N} .
$$

Replacing $\tilde{\Omega}_{k}$ by its expression using the matrices $\Gamma_{N}(k)$, $k=1, \ldots, N$ leads to $(6)$, which concludes the proof. $\diamond$

Remark 1 An interpretation of the B-L inequality in the context of robust analysis is provided in [7].

Remark 2 Considering the B-L inequality with $N=0$ allows retrieving the celebrated Jensen Inequality, which has been widely use in the literature. The case $N=1$ has been already proposed in [27]. The proof of this inequality was derived by application of the Wirtinger inequality. Unfortunately, this first approach did not suggest such a generic method to generate more inequalities. This corresponds to one of the main achievement of the present paper.

\section{Application to the stability analysis of sys- tems with a discrete delay}

\subsection{Systems with constant and known delay}

We present in this sub-section a first stability result for time-delay systems, which is based on the use of the B-L inequality developed in the previous section. The following stability theorem is provided by the use of Corollary 4 with an arbitrary $N$.

Theorem 5 For a given integer $N$ and a constant delay $h$, assume that there exist a matrix $P_{N} \in \mathbb{S}_{(N+1) n}$ and two matrices $S, R \in \mathbb{S}_{n}^{+}$such that the LMI

$$
\begin{aligned}
& \Theta_{N}(h)= \begin{cases}P_{N} \succ 0, & \text { if } N=0, \\
P_{N}+\frac{1}{h} \operatorname{diag}\left(0, S_{N-1}\right) \succ 0, & \text { if } N>0,\end{cases} \\
& \Phi_{N}(h)=\Phi_{N 0}(h)-\left[\begin{array}{c}
\Gamma_{N}(0) \\
\vdots \\
\Gamma_{N}(N)
\end{array}\right]^{T} R_{N}\left[\begin{array}{c}
\Gamma_{N}(0) \\
\vdots \\
\Gamma_{N}(N)
\end{array}\right] \prec 0,
\end{aligned}
$$

hold, where $\Gamma_{N}(k)$, for all $k=0, \ldots, N$, are defined in Corollary 4 and

$$
\begin{aligned}
& \Phi_{N 0}(h)=\operatorname{He}\left(G_{N}^{T}(h) P H_{N}\right)+\tilde{S}_{N}+h^{2} F_{N}^{T} R F_{N}, \\
& \tilde{S}_{N}=\operatorname{diag}\left\{S,-S, 0_{N n}\right\} \text {, } \\
& R_{N}=\operatorname{diag}\{R, 3 R, \ldots,(2 N+1) R\}, \\
& S_{N}=\operatorname{diag}\{S, 3 S, \ldots,(2 N+1) S\}, \\
& F_{N} \quad=\left[A A_{d} 0_{n, n N},\right] \text {, } \\
& G_{N}(h)=\left[\begin{array}{ccc}
I & 0_{n} & 0_{n, n N} \\
0_{n N, n} & 0_{n N, n} & h I_{n N}
\end{array}\right], \\
& H_{N}=\left[F_{N}^{T} \Gamma_{N}^{T}(0) \Gamma_{N}^{T}(1) \ldots \Gamma_{N}^{T}(N-1)\right]^{T} .
\end{aligned}
$$

Then the time delay system (1) is asymptotically stable for the constant delay $h$.

Proof: Guided by the B-L inequality (6) and the signals involved, we consider the following extra-states $\tilde{x}_{N}(t)$ defined by:

$$
\tilde{x}_{N}(t)=\left[\begin{array}{c}
x_{t}(0) \\
\int_{-h}^{0} L_{0}(s) x_{t}(s) \mathrm{d} s \\
\vdots \\
\int_{-h}^{0} L_{N-1}(s) x_{t}(s) \mathrm{d} s
\end{array}\right],
$$

if $N \geq 1$ and $\tilde{x}_{0}(t)=x_{t}(0)$, if $N=0$. The augmented vector $\tilde{x}_{N}$ is composed by the instantaneous state $x_{t}(0)$ and the projections of the state function $x_{t}$ to the $N$ first 
Legendre polynomials. Following the proof of Corollary 4 and equation (8), an integration by parts allows expressing the time derivative of $\tilde{x}_{N}$ as follows

$$
\dot{\tilde{x}}_{N}(t)=H_{N} \xi_{N}(t)
$$

where

$$
\xi_{N}(t)=\left[\begin{array}{c}
x_{t}(0) \\
x_{t}^{T}(-h) \\
\frac{1}{h} \int_{-h}^{0} L_{0}(s) x_{t}(s) \mathrm{d} s \\
\vdots \\
\frac{1}{h} \int_{-h}^{0} L_{N-1}(s) x_{t}(s) \mathrm{d} s
\end{array}\right], \quad N \geq 1
$$

and, if $N=0, \xi_{0}^{T}(t)=\left[x_{t}^{T}(0) x_{t}^{T}(-h)\right]$. It appears that this augmented system is the interconnection of the original delay system and a LTI system defined by the states $\int_{-h}^{0} L_{k}(s) x_{t}(s) \mathrm{d} s$, for $k=0, \ldots, N-1$. It is also worth mentioning that the only delayed term in (10) is $x_{t}(-h)$. Then, a natural choice for the LKF is

$$
\begin{aligned}
V_{N}\left(x_{t}, \dot{x}_{t}\right)= & \tilde{x}_{N}^{T}(t) P_{N} \tilde{x}_{N}(t)+\int_{t-h}^{t} x^{T}(s) S x(s) \mathrm{d} s \\
& +h \int_{t-h}^{t} \int_{\theta}^{t} \dot{x}^{T}(s) R \dot{x}(s) \mathrm{d} s \mathrm{~d} \theta,
\end{aligned}
$$

On a first hand, following the procedure provided in [11], the condition $S \succ 0$ allows applying Lemma 3 to the second term of $V_{N}$ to give a more accurate lower bound of the functional. In order to be consistent with the definition of $\tilde{x}_{N}$, Lemma 3 is considered with the order $N-1$. It thus yields

$$
\begin{aligned}
V_{N}\left(x_{t}, \dot{x}_{t}\right) \geq & \tilde{x}_{N}^{T}(t) \Theta_{N}(h) \tilde{x}_{N}(t) \\
& +h \int_{t-h}^{t} \int_{\theta}^{t} \dot{x}^{T}(s) R \dot{x}(s) \mathrm{d} s \mathrm{~d} \theta
\end{aligned}
$$

Then the positive definiteness of $V_{N}$ results from the conditions $S \succ 0, R \succ 0$ and $\Theta_{N}(h) \succ 0$. This also implies that there exists a sufficiently small $\epsilon_{1}>0$, such that $\Theta_{N}(h) \succ\left[\begin{array}{rr}\epsilon_{1} I & 0 \\ 0 & 0\end{array}\right]$. It follows that $V_{N}\left(x_{t}, \dot{x}_{t}\right) \geq \epsilon_{1}\left|x_{t}(0)\right|^{2}$. Furthermore, there exists a sufficiently large scalar $\lambda>0$ such that $P_{N} \prec \lambda \operatorname{diag}(I, I, 3 I, 5 I, \ldots,(2 N-1) I)$. It thus holds

$$
\begin{aligned}
V_{N}\left(x_{t}, \dot{x}_{t}\right) \leq & \lambda\left|x_{t}(0)\right|^{2}+\lambda \sum_{i=0}^{N-1}(2 i+1) \Omega_{i}^{T} \Omega_{i} \\
& +\int_{t-h}^{t} x^{T}(s) S x(s) \mathrm{d} s \\
& +h \int_{t-h}^{t} \int_{\theta}^{t} \dot{x}^{T}(s) R \dot{x}(s) \mathrm{d} s \mathrm{~d} \theta .
\end{aligned}
$$

Thanks to Lemma 3, we obtain

$$
\begin{aligned}
V_{N}\left(x_{t}, \dot{x}_{t}\right) \leq & \lambda\left|x_{t}(0)\right|^{2}+\int_{t-h}^{t} x^{T}(s)(\lambda h I+S) x(s) \mathrm{d} s \\
& +h \int_{t-h}^{t} \int_{\theta}^{t} \dot{x}^{T}(s) R \dot{x}(s) \mathrm{d} s \mathrm{~d} \theta,
\end{aligned}
$$

which guarantees that there exists a scalar $\epsilon_{2}>0$, such that $V_{N}\left(x_{t}, \dot{x}_{t}\right) \leq \epsilon_{2}\left|\bar{x}_{t}\right|_{h}^{2}$, for all $t>h$, where $\bar{x}_{t}=\left[\begin{array}{l}x_{t} \\ \dot{x}_{t}\end{array}\right]$. Then it holds

$$
\epsilon_{1}\left|x_{t}(0)\right|^{2} \leq V_{N}\left(x_{t}, \dot{x}_{t}\right) \leq \epsilon_{2}\left|\bar{x}_{t}\right|_{h}^{2} .
$$

Consider now the derivative of $V_{N}$, for all $t \geq h$. We obtain

$$
\begin{aligned}
\dot{V}_{N}\left(x_{t}, \dot{x}_{t}\right)= & 2 \tilde{x}_{N}^{T}(t) P_{N} \dot{\tilde{x}}_{N}(t)+x_{t}^{T}(0) S x_{t}(0) \\
& -x_{t}^{T}(-h) S x_{t}(-h)+h^{2} \dot{x}_{t}^{T}(0) R \dot{x}_{t}(0) \\
& -h \int_{-h}^{0} \dot{x}_{t}^{T}(s) R \dot{x}_{t}(s) \mathrm{d} s .
\end{aligned}
$$

By noting that $\tilde{x}_{N}(t)=G_{N}(h) \xi_{N}(t), \dot{\tilde{x}}_{N}(t)=H_{N} \xi_{N}(t)$ and $\dot{x}_{t}(0)=F_{N} \xi_{N}(t)$, it yields

$$
\dot{V}_{N}\left(x_{t}, \dot{x}_{t}\right)=\xi_{N}^{T}(t) \Phi_{N 0}(h) \xi_{N}(t)-h \mathcal{I}(\dot{x}, h)
$$

Finally, applying Corollary 4 to the order $N$ and injecting the resulting inequality into (14) leads to $\dot{V}_{N}\left(x_{t}, \dot{x}_{t}\right) \leq \xi_{N}^{T}(t) \Phi_{N}(h) \xi_{N}(t)$. Hence, if the LMI (9) are satisfied, there exists a scalar $\epsilon_{3}>0$ such that $\Phi_{N}(h) \prec\left[\begin{array}{cc}-\epsilon_{3} I & 0 \\ 0 & 0\end{array}\right]$. We finally obtain

$$
\dot{V}_{N}\left(x_{t}, \dot{x}_{t}\right) \leq-\epsilon_{3}\left|x_{t}(0)\right|^{2}, \quad \forall t \geq h .
$$

The end of the proof is taken from the proof of Theorem 1 from [5]. Integrating (15) we have

$$
V_{N}\left(x_{t}, \dot{x}_{t}\right)-V_{N}\left(x_{h}, \dot{x}_{h}\right) \leq-\epsilon_{3} \int_{h}^{t}\left|x_{s}(0)\right|^{2} d s
$$

and, hence, (12) yields

$$
\epsilon_{1}\left|x_{t}(0)\right|^{2} \leq V_{N}\left(x_{t}, \dot{x}_{t}\right) \leq V_{N}\left(x_{h}, \dot{x}_{h}\right) \leq \epsilon_{2}\left|\bar{x}_{h}\right|_{h}^{2} .
$$

Since $\left|x_{h}\right|_{h} \leq c_{1}|\phi|_{h}, c_{1}>0$ (cf. [12] p. 168) and $\dot{x}$, defined by the right-hand side of (1), satisfies $\left|\dot{x}_{h}\right|_{h} \leq$ $c_{2}|\phi|_{h}, c_{2}>0$, we obtain that

$$
\left|x_{t}(0)\right|^{2} \leq V_{N}\left(x_{h}, \dot{x}_{h}\right) / \epsilon_{1} \leq c_{3}|\phi|_{h}^{2}, c_{3}>0 .
$$

Hence, (1) is stable. To prove asymptotic stability we note that, for any initial condition $\phi, x$ is uniformly continuous on $[0, \infty)$ (since $\dot{x}$ defined by the right-hand side of (1) is uniformly bounded). Moreover, (16) yields that $\left|x_{t}(0)\right|^{2}$ is integrable on $[h, \infty)$. Then, by Barbalat's lemma, $x_{t}(0) \rightarrow 0$ as $t \rightarrow \infty$. Consequently, if the LMI of Theorem are satisfied, the delay system (1) is asymptotically stable for the constant delay $h$.

Remark 3 Taking $N=0$ in Theorem 5 allows retrieving one of the most classical delay-dependent stability conditions based on Jensen's inequality and LMI [9]. Additionally, choosing $N=1$ leads to the stability conditions from [27] 


\subsection{Remark on the choice of the LKF}

A comment on the LKF, $V_{N}$, and its relation with the class of functionals studied in $[10,25]$ is highlighted here. Indeed by considering the functional (11) and by defining the polynomial matrix $D(s)=\operatorname{diag}\left(0_{n}, L_{0}(s) I\right.$, $\left.L_{1}(s) I, \ldots, L_{N}(s) I\right)$ and the matrices

$$
\begin{aligned}
& \tilde{P}=\left[\begin{array}{c}
I \\
0_{n N, n}
\end{array}\right]^{T} P_{N}\left[\begin{array}{c}
I \\
0_{n N, n}
\end{array}\right], \quad S(s)=S \\
& Q(s)=\left[\begin{array}{c}
I \\
0_{n N, n}
\end{array}\right]^{T} P_{N} D(s), R(s, \xi)=D^{T}(s) P_{N} D(\xi) .
\end{aligned}
$$

Therefore, the functional $V_{N}$ can be rewritten as

$$
\begin{aligned}
V_{N}\left(x_{t}, \dot{x}_{t}\right)= & x^{T}(t) \tilde{P} x(t)+2 x^{T}(t) \int_{-h}^{0} Q(s) x_{t}(s) \mathrm{d} s \\
& +\int_{-h}^{0} \int_{-h}^{0} x_{t}^{T}(s) R(s, \xi) x_{t}(\xi) \mathrm{d} s \mathrm{~d} \xi \\
& +\int_{-h}^{0} x_{t}^{T}(s) S(s) x_{t}(s) \mathrm{d} s \\
& +h \int_{-h}^{0} \int_{\theta}^{0} \dot{x}_{t}^{T}(s) R \dot{x}_{t}(s) \mathrm{d} s \mathrm{~d} \theta
\end{aligned}
$$

The three first terms of $V_{N}$ are similar to the ones employed in [25] and in [10]. In [25], the degree of freedom comes from the degree of the polynomial matrices $Q(s)$, $S(s)$ and $R(s, \xi)$, denoted as $D_{p}$. In [10], the degree of the polynomial is always 1 but the degree of freedom comes from the degree of discretization, denoted latter on as $D_{d}$.

A first difference with respect to these two approaches is that in our setup, the polynomial matrix $S(s)$ is constant. The consequence is that our method requires less parameters to define the functional when increasing $D_{p}$ or $D_{d}$. Another difference relies on the last integral quadratic term of $V_{N}$ which depends on $\dot{x}_{t}$. Finally, the previous theorem does not need to enter into the sum of squares framework which generally requires the use of additional decision relaxation variables when testing the stability conditions.

\subsection{Delay range stability}

In Theorem 5, the delay is supposed to be perfectly known. Therefore, Theorem 5 only ensures the stability of the delay system for the pointwise delay $h$. This subsection extends this first result by considering that the delay $h$ is unknown but belongs to a prescribed interval $\left[h_{1}, h_{2}\right]$. We aim therefore at providing a criterion which ensures stability for all constant delays in this pocket.

Theorem 6 For a given integer $N$ and an uncertain constant delay $h \in\left[\begin{array}{ll}h_{1} & h_{2}\end{array}\right]$, assume that there exist a matrix $P_{N} \in \mathbb{S}_{(N+1) n}$ and $S, R \in \mathbb{S}_{n}^{+}$such that

$$
\Theta_{N}\left(h_{2}\right) \succ 0, \quad \Phi_{N}\left(h_{1}\right) \prec 0, \quad \Phi_{N}\left(h_{2}\right) \prec 0,
$$

hold, where $\Theta_{N}(h)$ and $\Phi_{N}(h)$ are defined in Theorem 5. Then the time delay system (1) is asymptotically stable for all constant delays $h$ in the interval $\left[h_{1}, h_{2}\right]$.

Proof : Assume that the LMI (18) hold and consider any delay $h$ in the interval $\left[h_{1}, h_{2}\right]$. We aim at proving that the two LMI conditions of Theorem 5 are satisfied of the delay $h$. On the first hand, the positive definiteness of $S$ directly implies that $\Theta_{N}(h) \succ \Theta_{N}\left(h_{2}\right)$, which thus guarantees the positive definiteness of $\Theta_{N}(h)$.

On the other hand, the application of the Schur Complement to the term $h^{2} F_{N}^{T} R F_{N}$ with the following decomposition $\left(h R F_{N}\right)^{T} R^{-1}\left(h R F_{N}\right)$ leads to an affine and, consequently convex LMI with respect to $h$. Then, this convexity property thus guarantees that

$$
\Phi_{N}(h) \preceq \frac{h-h_{1}}{h_{2}-h_{1}} \Phi_{N}\left(h_{2}\right)+\frac{h_{2}-h}{h_{2}-h_{1}} \Phi_{N}\left(h_{1}\right) .
$$

Finally the two last LMI of (18) ensure that the right hand side of the previous inequality is definite negative, which, by transition, ensures that $\Phi_{N}(h) \prec 0$.

\section{Hierarchy of LMI stability conditions}

This section is devoted to proving that the previous stability conditions form a hierarchy of LMI conditions. This is formulated in the following theorem based on the stability conditions of Theorem 5 .

Theorem 7 For any time delay system (1), define the set $\mathcal{H}_{N}$ by

$$
\mathcal{H}_{N}:=\left\{\begin{array}{r}
h \in \mathbb{R}^{+} \text {s.t. } \Theta_{N}(h) \succ 0, \quad \Phi_{N}(h) \prec 0, \\
P_{N}, S(N) \succ 0, R(N) \succ 0
\end{array}\right\}
$$

Then, it holds

$$
\mathcal{H}_{N} \subset \mathcal{H}_{N+1}, \quad \forall N \geq 0
$$

Proof : Let $N \in \mathbb{N}$. If $\mathcal{H}_{N}$ is empty, the inclusion is trivial. If $\mathcal{H}_{N}$ is not empty, then consider an element $h \in \mathcal{H}_{N}$. From the definition of $\mathcal{H}_{N}$, there exist symmetric matrices $P_{N}, S(N) \succ 0$ and $R(N) \succ 0$ such that $\Theta_{N}(h) \succ 0$ and $\Phi_{N}(h) \prec 0$. Taking advantages of the construction of the LKF (11), we suggest the matrices

$$
P_{N+1}=\left[\begin{array}{cc}
P_{N} & 0 \\
0 & \epsilon I
\end{array}\right],\left\{\begin{array}{l}
S(N+1)=S(N)=S \\
R(N+1)=R(N)=R
\end{array}\right.
$$


where $\epsilon$ is a scalar to be chosen. Then the matrix $\Theta_{N+1}(h)$ can be rewritten as

$$
\Theta_{N+1}(h)=\left[\begin{array}{cc}
\Theta_{N}(h) & 0 \\
0 & \frac{2 N+1}{h} S+\epsilon I
\end{array}\right] .
$$

Since $\Theta_{N}(h) \succ 0$ and $S \succ 0$, there exists a sufficiently small $\epsilon$ such that $\Theta_{N+1}(h) \succ 0$. Moreover it holds

$$
\begin{aligned}
& H_{N+1}=\left[\begin{array}{cc}
H_{N} & 0_{N n, n} \\
\Gamma_{N+1}(N)
\end{array}\right], \quad G_{N+1}(h)=\left[\begin{array}{cc}
G_{N}(h) & 0_{N n, n} \\
0_{n, N n} & h I
\end{array}\right], \\
& \tilde{S}_{N+1}=\left[\begin{array}{cc}
\tilde{S}_{N} & 0_{N n, n} \\
0_{n, N n} & 0_{n}
\end{array}\right], \quad F_{N+1}=\left[\begin{array}{ll}
F_{N} & 0_{n}
\end{array}\right] .
\end{aligned}
$$

From these expressions, the matrix $\Phi_{N+1}(h)$ can be expressed using the matrix $\Phi_{N}(h)$ as follows

$$
\begin{aligned}
\Phi_{N+1}(h) & =\left[\begin{array}{cc}
I & 0 \\
\Gamma_{N+1}(N+1)
\end{array}\right]^{T}\left[\begin{array}{cc}
\Phi_{N}(h) & 0 \\
0 & -(2 N+3) R
\end{array}\right] \\
& \times\left[\begin{array}{cc}
I & 0 \\
\Gamma_{N+1}(N+1)
\end{array}\right]+\epsilon H e\left\{h \Gamma_{N+1}^{T}(N)\left[\begin{array}{l}
0 \\
I
\end{array}\right]\right\} .
\end{aligned}
$$

Since $\Phi_{N}(h) \prec 0, R \succ 0$ and by noting that the matrix $\left[\begin{array}{cc}I & 0 \\ \Gamma_{N+1}(N+1)\end{array}\right]$ is non singular, the first term of the previous expression is negative definite. It implies that there exists a sufficiently small $\epsilon$ for which $\Phi_{N+1}(h) \prec$ 0 and $\Theta_{N+1}(h) \succ 0$. Therefore, $h$ belongs to $\mathcal{H}_{N+1}$. Finally, since $h$ is any element of $\mathcal{H}_{N}$, it implies that $\mathcal{H}_{N} \subset \mathcal{H}_{N+1}$.

Theorem 7 proves that, the stability conditions provided in Theorem 5 at the order $N+1$ delivers, at least, the same result the same condition taken at the order $N$. Moreover, since Theorem 5 only provides sufficient stability condition, the set $\mathcal{H}_{N}$, for a given $N \in \mathbb{N}$ represents an inner approximation of the stability pockets. However, the previous theorem does not prove that the conditions of Theorem 5 will converge to the analytical bounds of the delay. A similar development shows that Theorem 6 also forms a hierarchy of stability conditions.

\section{Examples}

The purpose of the following section is to illustrate on academic and non trivial examples how the inequalities given in Section 3 lead to a relevant reduction of conservatism in the stability condition.

\subsection{Example 1}

Consider the linear time-delay system (1) with the matrices

$$
A=\left[\begin{array}{cc}
-2 & 0 \\
0 & -0.9
\end{array}\right], \quad A_{d}=\left[\begin{array}{cc}
-1 & 0 \\
-1 & -1
\end{array}\right]
$$

This system is a well-known delay dependent stable system, that is the delay free system is stable and the maximum allowable delay $h_{\max }=6.1725$ can be easily computed by delay sweeping techniques. The results are reported in Table 1 . Many recent papers give the same result since they are intrinsically based on the same LKF and use the same bounding cross terms technique i.e. Jensen inequality. Some papers [31],[30] which use an augmented Lyapunov, based on the addition on a triple Integral term on the LKF can go further but with a numerically increasing burden, compared to our proposal. The partitioning approach proposed by [13] based on the discrete delay decomposition gives an upperbound which tends to the analytical value even if the numerical complexity remains important. The robust approach [16] gives a very good upper-bound with a similar computational complexity than our present result. The discretized LKF proposed by [10] as well as the sum of square optimization scheme developed by Peet et al [25] give a delay upperbound very closed to the maximum allowable delay with an increasing numerical complexity.

\subsection{Example 2}

This example is provided to illustrate Theorem 7. Note that this system has not been studied in the literature of time delay system using the Lyapunov-Krasovskii Theorem and LMI conditions. It is extracted from the dynamics of machining chatter $[32,29]$ and is given by

$$
\left\{\begin{array}{l}
\dot{x}(t)=A x(t)+B u(t) \\
y(t)=C x(t)
\end{array}\right.
$$

with

$$
A=\left[\begin{array}{cccc}
0 & 0 & 1 & 0 \\
0 & 0 & 0 & 1 \\
-10 & 10 & 0 & 0 \\
5 & -15 & 0 & -0.25
\end{array}\right], \quad B=\left[\begin{array}{l}
0 \\
0 \\
1 \\
0
\end{array}\right], \quad C=\left[\begin{array}{l}
1 \\
0 \\
0 \\
0
\end{array}\right]^{T}
$$

A delayed static output feedback controller is proposed:

$$
u(t)=-K y(t)+K y(t-h),
$$

where $K$ is the gain of the controller and $h$ is an unknown constant delay. The resulting dynamics is thus modeled 


\begin{tabular}{|c|c|c||c|c|c|}
\hline Theorems & $h_{\max }$ & number of variables & Theorems & $h_{\max }$ & number of variables \\
\hline \hline$[9,14,28,31]$ & 4.472 & $1.5 n^{2}+1.5 n$ & {$[10], D_{d}=1$} & 6.053 & $7.5 n^{2}+3.5 n$ \\
{$[16]$} & 6.1107 & $1.5 n^{2}+9 n+9$ & {$[10], D_{d}=2$} & 6.165 & $10.5 n^{2}+4.5 n$ \\
{$[2]$} & 5.120 & $7 n^{2}+4 n$ & {$[10], D_{d}=3$} & 6.171 & $14.5 n^{2}+4.5 n$ \\
{$[31]$} & 5.02 & $18 n^{2}+18 n$ & {$[10], D_{d}=4$} & 6.171 & $20.5 n^{2}+5.5 n$ \\
{$[18]$} & 4.97 & $69 n^{2}+5 n$ & {$[25], D_{p}=1$} & 5.19 & $7 n^{2}+3 n$ \\
{$[1]$} & 5.120 & $6.5 n^{2}+3.5 n$ & {$[25], D_{p}=2$} & 5.90 & $12.5 n^{2}+4.5 n$ \\
{$[30]$} & 5.30 & $8.5 n^{2}+3.5 n$ & {$[25], D_{p}=3$} & 6.10 & $21 n^{2}+6 n$ \\
{$[13], D_{d}=2$} & 5.71 & $4 n^{2}+2 n$ & Th.5, $N=0$ & 4.472 & $1.5 n^{2}+1.5 n$ \\
{$[13], D_{d}=3$} & 5.96 & $6.5 n^{2}+2.5 n$ & Th.5, $N=1$ & 6.059 & $3 n^{2}+2 n$ \\
{$[13], D_{d}=4$} & 6.05 & $10 n^{2}+3 n$ & Th.5, $N=2$ & 6.168 & $5.5 n^{2}+2.5 n$ \\
{$[26]$} & 5.901 & $3 n^{2}+2 n$ & Th. $5, N=3$ & 6.1725 & $9 n^{2}+3 n$ \\
\hline
\end{tabular}

Table 1

Results for Example (19) for constant delay $h$. The degree of discretization $D_{d}$ and the degree of the polynomial $D_{p}$ are defined in subsection 4.2 .

$h$

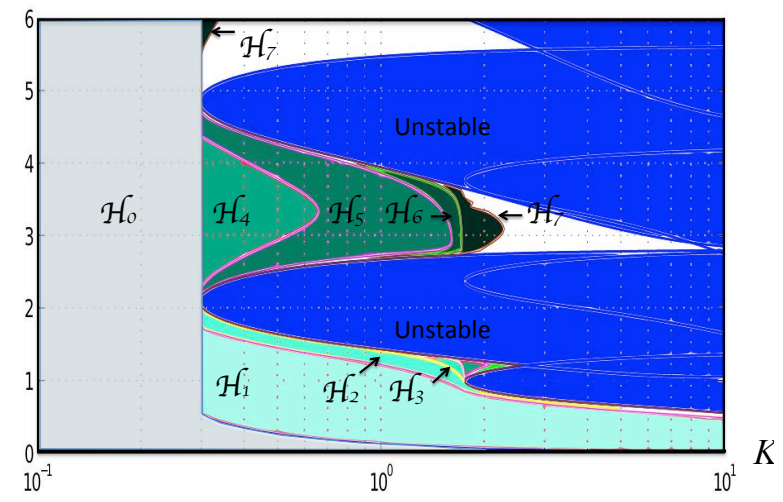

Fig. 1. Stability region in the plan $(K, h)$, obtained using Theorem 5 for $N=0, \ldots, 7$.

by a time-delay system:

$$
\dot{x}(t)=A_{0} x(t)+A_{1} x(t-h),
$$

with $A_{0}=A-B K C$ and $A_{1}=B K C$. The results are summarized in Figure 1 which shows the stability regions in the $(K, h)$ plane. The blue region represents the instability region which have been calculated using a griding over $K$ along with the allmargin function of the Control Toolbox of Matlab(c). Then, Theorem 5 provides inner approximations of the stability region delimited by colored curves. The curve $N=0$ perfectly detects the independent of the delay stability region (for $K \leq 0.3$ ) as well as a first delay dependent stability pocket. It corresponds to the maximal allowable delay when Jensen's Lemma is used when establishing the stability criterion. Taking $N=1$ allows retrieving the same results as in [27] which uses Wirtinger's Lemma. Clearly, increasing $N(N=0,1, \ldots, 7)$ allows reducing the pessimism and discovering new stability pockets. This figure illustrates the implication of Theorem 7 on the inclusions $\mathcal{H}_{0} \subset \mathcal{H}_{1} \subset \cdots \subset \mathcal{H}_{7}$. Another important remark is that increasing $N$ can improve significantly the inner approximations of the stability region. For instance, $\mathcal{H}_{4} \backslash \mathcal{H}_{3}$, $\mathcal{H}_{5} \backslash \mathcal{H}_{4}$ or even $\mathcal{H}_{7} \backslash \mathcal{H}_{6}$ are surprisingly large sets.

\section{Conclusions}

In this paper, we have provided a new set of integral inequalities which encloses the well known Jensen inequality, widely used in the stability study of time delay systems. It is based on the extensive use of Legendre polynomials. These inequalities are the source of a new proposal for Lyapunov functions. A stability analysis driven by these inequalities leads to a set of LMI criteria which are highly efficient at least on examples in terms of conservatism and complexity. We have also proved that the set of stability conditions forms a hierarchy of LMI indexed by the polynomial degree $N$, in the sense that increasing $N$ reduces the conservatism of the proposed method. Future works will include the study of the asymptotic necessity of this approach and also the case of time-varying delays.

\section{References}

[1] Y. Ariba and F. Gouaisbaut. An augmented model for robust stability analysis of time-varying delay systems. Int. Journal Control, 82(9):1616-1626, 2009.

[2] Y. Ariba, F. Gouaisbaut, and K.H. Johansson. Stability interval for time-varying delay systems. In 49th IEEE Conference on Decision and Control (CDC'10), pages $1017-1022,2010$.

[3] C. Briat. Convergence and equivalence results for the Jensen's inequality - application to time-delay 
and sampled-data systems. IEEE Trans. on Automatic Control, 56(7):1660-1665, 2011.

[4] E. Fridman. New Lyapunov-Krasvoskii functionals for stability of linear retarded and neutral type systems. Systems and Control Letters, 43:309-319, 2001.

[5] E. Fridman, A. Seuret, and J.-P. Richard. Robust sampled-data stabilization of linear systems: An input delay approach. Automatica, 40(8):1141-1446, 2004.

[6] E. Fridman and U. Shaked. A descriptor system approach to $H^{\infty}$ control of linear time-delay systems. IEEE Trans. on Automatic Control, 47(2):253-270, 2002.

[7] F. Gouaisbaut, Y. Ariba, and A. Seuret. Bessel inequality for robust stability analysis of time-delay system. In 52nd IEEE Conference on Decision and Control (CDC'13), 2013.

[8] F. Gouaisbaut and D. Peaucelle. Delay-dependent stability analysis of linear time delay systems. In IFAC Workshop on Time Delay System (TDS'06), Aquila, Italy, July 2006.

[9] F. Gouaisbaut and D. Peaucelle. A note on stability of time delay systems. In $5^{\text {th }}$ IFAC Symposium on Robust Control Design (ROCOND'06), Toulouse, France, 2006.

[10] K. Gu. A further refinement of discretized Lyapunov functional method for the stability of timedelay systems. Int. Journal of Control, 74(10):967 - 976, 2001.

[11] K. Gu, V. L. Kharitonov, and J. Chen. Stability of Time-Delay Systems. Birkhäuser Boston, 2003. Control engineering.

[12] J. Hale and S. M.V. Lunel. Introduction to Functional Differential Equations. Springer-Verlag, New York, 1993.

[13] Q.-L. Han. A discrete delay decomposition approach to stability of linear retarded and neutral systems. Automatica, 45(2):517 - 524, 2009.

[14] Y. He, Q. G. Wang, L. Xie, and C. Lin. Further improvement of free-weighting matrices technique for systems with time-varying delay. IEEE Trans. on Automatic Control, 52(2):293-299, 2007.

[15] X. Jiang and Q.L. Han. Delay-dependent robust stability for uncertain linear systems with interval time-varying delay. Automatica, 42(6):1059-1065, 2006.

[16] C.Y. Kao and A. Rantzer. Stability analysis of systems with uncertain time-varying delays. Automatica, 43(6):959 - 970, 2007.

[17] V.L. Kharitonov and A. Zhabko. LyapunovKrasovskii approach to the robust stability analysis of time-delay systems. Automatica, 39(1):15-20, 2003.

[18] J.H. Kim. Note on stability of linear systems with time-varying delay. Automatica, 47(9):2118-2121, 2011.

[19] V.B. Kolmanovskii and J.-P. Richard. Stability of some linear systems with delay. IEEE Trans. on
Automatic Control, 44(5):984-989, 1999

[20] K. Liu and E. Fridman. Wirtinger's inequality and Lyapunov-based sampled-data stabilization. Automatica, 48(1):102-108, 2012.

[21] S. Mondie, V.BL. Kharitonov, and O. Santos. Complete Lyapunov-Krasovskii functional with a given cross term in the time derivative. In $44^{\text {th }}$ IEEE Conference on Decision and Control (CDC'05), Sevilla, Spain, 2005.

[22] S.-I. Niculescu. Delay Effects on Stability. A Robust Control Approach. Springer-Verlag, 2001.

[23] P. Park, J.W. Ko, and C. Jeong. Reciprocally convex approach to stability of systems with timevarying delays. Automatica, 47(1):235-238, 2011.

[24] M.M. Peet and P.-A. Bliman. On the conservatism of the sum-of-squares method for analysis of timedelayed systems. Automatica, 47(11):2406-2411, 2011.

[25] M.M. Peet, A. Papachristodoulou, and S. Lall. Positive forms and stability of linear time-delay systems. SIAM Journal on Control and Optimization, 47(6):3227-3258, 2009.

[26] A. Seuret and F. Gouaisbaut. On the use of the Wirtinger's inequalities for time-delay systems. In $10^{\text {th }}$ IFAC Workshop on Time Delay Systems (IFAC TDS'12), Boston, MA, USA, 2012.

[27] A. Seuret and F. Gouaisbaut. Wirtinger-based integral inequality: Application to time-delay systems. Automatica, 49(9):2860-2866, 2013.

[28] H. Shao. New delay-dependent stability criteria for systems with interval delay. Automatica, 45(3):744749, 2009.

[29] R. Sipahi, S. Niculescu, C.T. Abdallah, W. Michiels, and K. Gu. Stability and stabilization of systems with time delay. Control Systems, IEEE, 31(1):38-65, 2011.

[30] J. Sun, G.P. Liu, and J. Chen. Delay-dependent stability and stabilization of neutral time-delay systems. International Journal of Robust and Nonlinear Control, 19(12):1364-1375, 2009.

[31] J. Sun, G.P. Liu, J. Chen, and D. Rees. Improved delay-range-dependent stability criteria for linear systems with time-varying delays. Automatica, 46(2):466 - 470, 2010.

[32] J. Zhang, C. R. Knopse, and P. Tsiotras. Stability of time-delay systems: Equivalence between Lyapunov and scaled small-gain conditions. IEEE Trans. on Automatic Control, 46(3):482-486, March 2001. 